\section{Natürliche Erkältungsmedizin}

Wenn Babys husten, wünschen sich Eltern schnelle und sanfte Hilfe. Eine Behandlungsoption, auch schon für die ganz kleinen Patienten ab dem sechsten Monat, bietet die natürliche Phytotherapie von Eucabal ${ }^{\oplus}$ mit der Kombination aus Balsam $S$ und Hustensaft.

Zum „Wegstreicheln“ von Erkältungen eignet sich das milde Eucabal ${ }^{\circledR}$-Balsam S. Diese pflanzliche Creme kombiniert wertvolles Eukalyptus- und Kiefernnadelöl - und enthält bewusst kein Campher, Menthol oder Konservierungsstoffe. Zugelassen ist Eucabal ${ }^{\oplus}$-Balsam S schon für Babys ab sechs Monate zur Einreibung auf dem Rücken. Es ist bis zum zwölften Lebensjahr verordnungsfähig.

Als Begleitmedikation empfiehlt sich Eucabal ${ }^{\circledR}$-Hustensaft. Dieser ebenfalls pflanzliche Hustenlöser entfaltet mit seiner einzigartigen Kombination aus Thymian- und Spitzwegerich-Fluidextrakt eine doppelt antibakterielle Wirkungskraft: Die adstringierenden Inhaltsstoffe hüllen die strapazierte Bronchialschleimhaut ein und schützen sie auf diese Weise vor zusätzlichem Bakterienbefall. Durch seine krampflösenden und expektorierenden Eigenschaften lindert der Hustensaft den Hustenreiz, verflüssigt zähen Bronchialschleim und fördert damit das produktive Abhusten.

Nach Informationen von esparma Arzneimittel

\section{Schnelle Hilfe bei Verstopfung}

Verstopfung ist keine Frage des Alters: Bereits Säuglinge und Kleinkinder leiden vermehrt unter Obstipation. Neben der Änderung von Ernährung und Lebensgewohnheiten kann auch der Einsatz eines osmotischen Laxans indiziert sein. Hier empfiehlt die aktuelle Leitlinie der GPGE bei initialer oder akuter Obstipation bei Säuglingen Microklist ${ }^{\circledast}$, das seit August 2012 unter dem Namen Microlax ${ }^{\circledR}$ erhältlich ist. Microlax ${ }^{\circledast}$ kann bei Kindern und Säuglingen angewendet werden und ist auch für Schwangere zugelassen.

Innerhalb von nur fünf bis 20 Minuten kommt es zu einer sicheren und planbaren Erleichterung, die einem normalen Stuhlgang gleicht. Der gelartige Inhalt der Mini-Tube wirkt rein lokal. Bei der Anwendung kommt es zu keiner Resorption. Außer möglichen Überempfindlichkeitsreaktionen sind keine systemischen $\mathrm{Ne}$ benwirkungen bekannt, ebenso keine Gewöhnungseffekte oder Abhängigkeiten.

Nach Informationen von Johnson \& Johnson

\title{
Meningokokken-Impfstoff für Kinder ab einem Jahr
}

— Die von Meningokokken ausgelöste Meningitis oder Sepsis sind lebensbedrohliche Krankheiten, die bei einem fulminanten Verlauf innerhalb von 24 bis 48 Stunden zum Tod führen können. Insbesondere Reisende in die Subsahara bzw. den „Meningitisgürtel“ Afrikas sind einem höheren Risiko ausgesetzt. „Hier treten häufiger Epidemien auf, bei welchen vor allem die Serogruppe A dominiert. Gerade in abgelegenen Gebieten kann es ein Problem sein, schnell genug an ärztliche Hilfe zu kommen", betonte die reisemedizinische Expertin Prof. Annelies Wilder-Smith, Heidelberg. Auch bei großen Menschenansammlungen, wie der Pilgerfahrt nach Mekka, kam es in den vergangenen Jahren immer wieder zu Ausbrüchen, insbesondere durch die Serogruppe $W_{135}$.

Die Europäische Arzneimittelagentur (EMA) hat nun einen tetravalenten Konjugatimpfstoff (Nimenrix ${ }^{\top M}$ ) zugelassen, der mit A, C, $W_{135}$ und $Y$ vier der fünf häufigsten Serogruppen von Neisseria meningitidis abdeckt. Neu ist, dass bereits Kleinkinder ab dem vollendeten ersten Lebensjahr mit einer Einmaldo- sis geimpft werden können. Der an ein Tetanustoxoid gekoppelte Impfstoff zeigt im Vergleich zu Polysaccharidimpfstoffen eine verbesserte Antikörperantwort. Er eignet sich zudem als Booster-Impfstoff, da Personen, die zuvor eine Impfung mit Polysaccharid erhielten, ebenfalls mit einer höheren Immunantwort reagierten.

Zugleich lassen sich bei Erwachsenen weitere Reiseimpfstoffe (etwa Hepatitis A, B) und bei Kindern die meisten Standardimpfungen (Masern-Mumps-Röteln und Varizellen) parallel applizieren. Die Verträglichkeit ist im Allgemeinen gut, als häufigste Nebenwirkungen traten etwa Appetitlosigkeit und Fieber sowie lokale Rötungen oder Schwellungen auf. Die aktive Impfung kann Kleinkindern, Kindern und Jugendlichen für mindestens zwei bzw. bis zu dreieinhalb Jahren Schutz gegen Meningokokken bieten.

Dr. Marion Hofmann-Aßmus

Satelliten-Symposium „Prevention of meningococcal disease in travelers"; Dublin/Irland, 7. Juni 2012. Veranstalter: GlaxoSmithKline

\section{Effektiv gegen schmerzhaften Husten und Bronchitis}

— Schmerzhafter Husten, festsitzender Schleim und enge Bronchien, die das Atmen erschweren - akute Infektionen der Atemwege gehören zu den häufigsten Beratungsanlässen in der Arztpraxis. Wichtiges Therapieziel bei akutem Husten und Bronchitis ist die rasche Linderung der z.T. starken Beschwerden. Gleichzeitig wünschen sich immer mehr Patienten nebenwirkungsarme Alternativen zu chemisch-synthetischen Mitteln. Das pflanzliche Kombinationspräparat Bronchipret ${ }^{\circledR}$ vereint beide Vorteile.

Bronchipret $^{\oplus}$, die Pflanzenkombination aus Thymian und Efeu oder Primel, ist besonders effektiv bei der Behandlung des akuten Hustens und der Bronchitis, löst den festsitzenden Schleim, heilt die Entzündung in den Bronchien und reduziert die Zahl der Hustenanfälle. Die Bronchien entspannen sich und die Patienten können besser durchschlafen. Die Heilpflanzen Thymian und Efeu bzw. Primel ergänzen sich in der Zweierkombination des Medikaments durch ihre sekretnormalisierenden, antientzündlichen, bronchospasmolytischen und antimikrobiellen Eigenschaften. Zudem wirken die Pflanzenextrakte synergistisch, d. h. sie verstärken sich gegenseitig. Gleichzeitig ist das Phytotherapeutikum gut verträglich und nebenwirkungsarm.

Zwei placebokontrollierte klinische Studien konnten eine Reduktion des Hustens und einen Rückgang der Bronchitis-Symptome nachweisen. Bronchipret ${ }^{\circledR}$ wird als einziges Therapeutikum in der aktuellen S3-HustenLeitlinie der Deutschen Gesellschaft für Pneumologie und Beatmungsmedizin bei akutem Husten empfohlen.

Nach Informationen von Bionorica 